\title{
Long QT Syndrome
}

National Cancer Institute

\section{Source}

National Cancer Institute. Long QT Syndrome. NCI Thesaurus. Code C34786.

A ventricular arrhythmia characterized by a long QT interval, and accompanied by syncopal episodes sometimes leading to sudden death due to paroxysmal ventricular arrhythmia. This arrhythmia is associated with a prolong ation of repolarization following depolarization of the cardiac ventricles. The prolong ation of the Q-T interval combined with torsades de pointes manifests as several different forms; some may be acquired or congenital; some may lead to serious arrhythmia and sudden cardiac death. 